\title{
Multiple primary cranio-spinal tumours in a 13-year-old female with neurofibromatosis type 2 management strategy
}

\author{
Teresa Stachowicz-Stencel • Anna Synakiewicz • \\ Ewa Bien • Elzbieta Adamkiewicz-Drozynska • \\ Miroslawa Wybieralska-Dubaniewicz • Anna Balcerska
}

Received: 29 January 2010 / Accepted: 15 July 2010 /Published online: 27 July 2010

(C) The Author(s) 2010. This article is published with open access at Springerlink.com

\begin{abstract}
Introduction Neurofibromatosis type 2 (NF2) is an inherited, rare autosomal dominant syndrome characterised by the development of multiple benign cranial and spinal tumours, peripheral neuropathy, ophthalmological and cutaneous lesions. Herein, we report one case of NF2 treated with multivariate chemotherapy.

Material and methods A 13-year-old female presented with multiple cranio-spinal tumours in MRI. First symptoms were progressive changes in vision, left-sided paresis, unilateral sensorineural hearing loss, and left hypoglossal nerve paresis. The patient underwent palliative, partial surgical resection of the tumour which was located in a posterior fossa. Histopathological examination showed a psammomatous meningioma located near the great foramen and schwannomas of VIII nerve in the cerebello-pontine angle. Clinical and radiological examination revealed a rapid progression of the disease. As such, multivariate chemotherapy was used. The patient died 4 years after diagnosis.

Conclusion NF2 patients with multiple tumours at diagnosis may not be treatable with surgery alone and, as a result,
\end{abstract}

T. Stachowicz-Stencel $(\bowtie) \cdot A$. Synakiewicz $\cdot$ E. Bien

E. Adamkiewicz-Drozynska $\cdot$ A. Balcerska

Department of Paediatrics, Haematology,

Oncology and Endocrinology, Medical University of Gdansk,

7 Debinki Street,

80-211 Gdansk, Poland

e-mail: tsten@gumed.edu.pl

M. Wybieralska-Dubaniewicz

Department of Radiology, Medical University of Gdansk,

Gdansk, Poland presentation with such a disease in childhood results in poor prognosis. The unification of management strategies in NF2 patients is highly desirable.

Keywords Children · Cranio-spinal tumours . Neurofibromatosis type 2. Prognosis · Treatment

\section{Introduction}

Neurofibromatosis type 2 (NF2) is an inherited, rare autosomal dominant syndrome characterized by the development of multiple benign cranial and spinal tumours (schwannomas, meningiomas, ependymomas), peripheral neuropathy, ophthalmological and cutaneous lesions [1-3]. Tumours associated with NF2 are caused by the inactivation of both alleles of the NF2 tumour-suppressor gene that has been localised on the chromosome 22q12, by mutation or allele loss [1-5]. Recent evidence suggests that between $20 \%$ and $30 \%$ of NF2 cases without a family history of the disease are mosaic for the underlying disease-causing mutation and more than $50 \%$ of patients represent new mutations [4]. The disorder appears in one of 25,000 live births [1]. About $10 \%$ of NF2 patients are under the age of 10 years old. Among patients with NF2, $18 \%$ present with symptoms of the disease at the age of 15 years or less [6]. Although most tumours in the central nervous system in NF2 are slow growing, they may grow faster during childhood. In this situation, the prognosis is often poor [7]. The development of new tumours is likely in patients with NF2, even after surgery $[4,5]$. In these situations, a new management strategy and early diagnosis are necessary. 
In this report, we describe the treatment of a 13-year-old female with NF2 who had multiple types of primary tumours in the brain and spinal canal.

\section{Case report}

A 13-year-old female was admitted to the Department of Paediatric Oncology because of complaints of progressive changes in vision, left-sided paresis, unilateral sensorineural hearing loss, left hypoglossal nerve paresis and difficulties in learning at school over a period of 6 months. The physical examination showed one café-au-lait spot on the left upper leg (greater than $10 \mathrm{~cm}$ in diameter) and one on the left scapula, schwannomas of the forearms and dysmorfic face (depressed nasal root, hypertelorism, small and downward slanting palpebral fissures, short philtrum with a tented upper lip and mandibular hypoplasia). Neurological examination revealed bilateral Babinski's reflex, left-sided muscular atrophy and a deflection of the tongue to the left. Magnetic resonance imaging (MRI) of the brain and spinal canal revealed multiple cranial nerves tumours: V-trigeminal nerve, bilateral vestibular schwannomas and right-sided meningioma, and meningioma in the area of the optic nerve (Fig. 1a, b). In the posterior cranial fossa, the huge focus involving the foramen magnum was described as schwannomas of VIII nerve (Fig. 2). Multiple spinal canal nodules, involving L5-S1 level, were observed (Fig. 3). The patient underwent palliative, partial surgical resection of the posterior fossa tumour. Histopathological examination showed psammomatous meningioma localised near the foramen magnum and schwannoma in the cerebello-pontine angle.

Following the operation, an increased paresis of lower limbs and neurogenic bladder was observed. The excision of the Th11-Th12 tumour was performed. Histopathology proved meningioma. Staphylococcus sepsis and acute renal failure were the side effects after surgery.

She finished the treatment with clinical success.

Fourteen months later, progression of the disease was stated. Bilateral deafness, facial nerve paresis, a deflection of the tongue to the right, left eye-ball oedema, visual disorders and ape hand were all observed.

She received chemotherapy (vinblastin $6 \mathrm{mg} / \mathrm{m}^{2}$ every 2 weeks for 3 months). In the course of performing MRI of the brain and spinal canal, progression of the disease was observed. The general condition of the patient deteriorated. She was presented with difficulties in talking and severe coordination disturbances; she was unable to walk in a straight line and had lost the ability to manipulate small objects. The chemotherapy for low-grade gliomas protocol, according to the Memorial Hospital for Children in Warsaw, was administered thereafter. It was comprised of: intensive chemotherapy-carboplatin $\left(550 \mathrm{mg} / \mathrm{m}^{2} ; 1,4,7,10\right.$ weeks), vincristin $\left(1.5 \mathrm{mg} / \mathrm{m}^{2}, 1-10\right.$ week) and maintenance chemotherapy-carboplatin with vincristin every 4 weeks.

After 6 months, no response to chemotherapy was stated. Due to no possibility to remove the tumour radically, the child was included in a clinical trial. She received Irinotecan in doses of $20 \mathrm{mg} / \mathrm{m}^{2}$ for 10 days every 3 weeks. In all, nine cycles were administered to the patient. Thanks to Irinotecan, she achieved the clinical stagnation of the disease.

Following a period of 2 years, progression of the disease was once more present. She was treated with temozolomide $\left(150 \mathrm{mg} / \mathrm{m}^{2}\right)$ for 3 months. Finally, our patient died of the progression of the disease 4 years after diagnosis.

\section{Discussion}

NF2 is an uncommon genetic disorder, which is characterized by an increased risk of benign nervous system tumour development [8]. The diagnostic criteria for neurofibromatosis type 2 is bilateral vestibular schwannomas (VS) or family history of NF2 according to first degree family relative, unilateral vestbular schwannomas under 30 years old, or any two of the following: meningioma, glioma, schwannoma, juvenile posterior subcapsular lenticular opacities/juvenile cortical cataract [9-12]. Nunes et al. [13], presented clinical data on 12 patients with NF2 at age before 18 years and in large number with positive family history. One third of the children had hearing impairments and two thirds presented with dysfunctions of the cranial nerve. Radiological examinations revealed cranial meningiomas in $75 \%$ of cases, cranial schwannoms in $83 \%$, and spinal cord tumours in $75 \%$. Evans et al. [14] showed that at least $18 \%$ of NF2 sufferers presented in childhood with isolated features of the disease had no family history. These paediatric patients presented with a more severe course of the disease with multiple tumours. In the study of Bosch et al. [15], children with early onset of the disease presented with ophthalmologic symptoms and lower motor neuron extremity weakness and in those with late disease onset, eight nerve impairment was observed. MacCollin and Mautner [16] showed that the first ocular manifestation of NF2 in the paediatric population was visual loss or diplopia. Our patient had an atypical onset of this autosomal-dominant disorder. After a few months, she presented with hearing loss, most often related to the development of vestibular schwannomas. Hearing loss, often accompanied by tinnitus, occurs in around $60 \%$ of adults and up to $30 \%$ of children $[17,18]$. The MRI scan showed the presence of tumours in the central nervous system and spinal canal. In the literature, there are a great many reports confirming the variety of spinal tumours associated with NF2 [2, 19, 20]. Bilateral vestibular 
Fig. 1 a Contrast-enhanced conventional T1-weighted MR image: neuromas of the $\mathrm{V}$ and VIII nerve and sagittal right-sided meningioma. b Contrast-enhanced transversal T1-weighted MR image: the optic nerve glioma

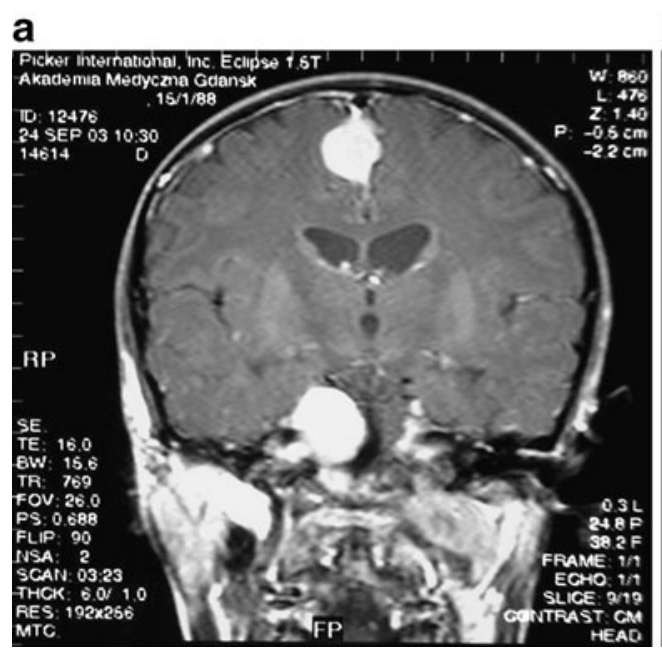

b

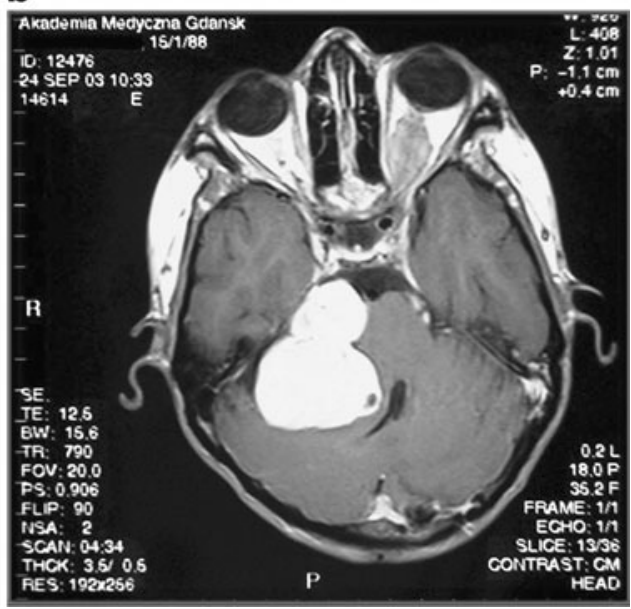

schwannomas are found in $90-95 \%$ of patients with NF2. It is reported that more than $99 \%$ of VS in NF2 are benign, but they remain an important cause of mortality due to their location [1]. Schwannomas can develop along the course of cranial, spinal and peripheral nerves, differently than vestibular. Most often it arises from the oculomotor, trigeminal and facial nerves [21]. Schwannomas of spinal nerves may result in discrete peripheral neuropathies. In our patient, the histopathology examination showed meningioma in the posterior cranial fossa, which is the second most common tumour connected with NF2. Intracranial meningiomas appear in $45-58 \%$ of patients with this disorder and intradural extramedullary spinal meningiomas in $20 \%$ [2]. Although meningiomas are benign, they may give clinical symptoms, the nature of which are related to their size and anatomical location. It is proven that meningiomas associ-

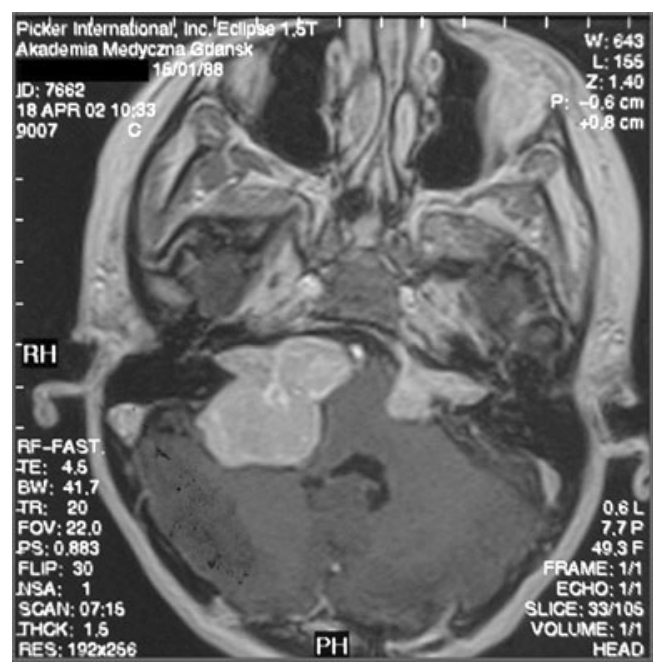

Fig. 2 In the posterior cranial fossa, bilateral pathological solid masses located in pontocerebellar cisterns, which extend to the auditory meatus; intensive contrast enhancement MR imageschwannomas of the VIII nerve

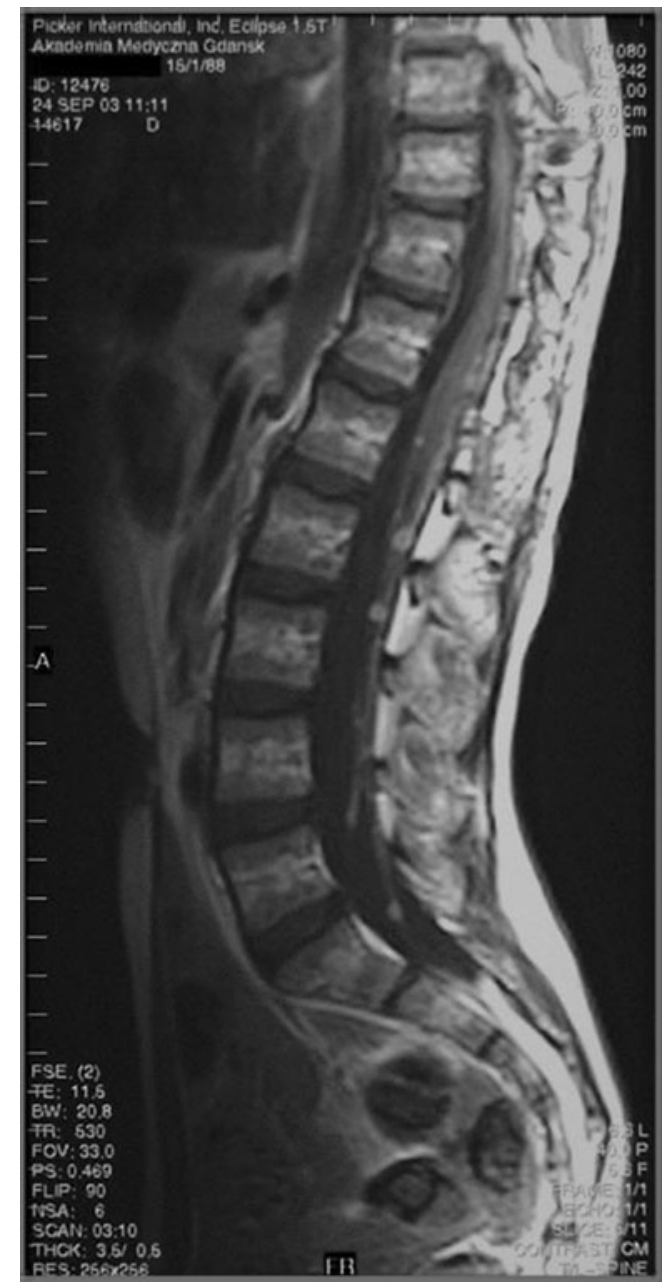

Fig. 3 Contrast-enhanced T1-weighted MR image: numerous intradural spinal canal nodules and small tumours in intervertebral foramen on the right side at level L5-S1 
ated with NF2 more frequently have higher proliferative activity and a tendency to form more atypical and anaplastic grades than sporadic meningiomas [22].

The typical treatment strategy for patients with VS is "watch and wait then rescan", complete with surgical resection and stereotactic radiotherapy. The authors noted that more than $50 \%$ of VS were stable in size, so in this situation the "watch and wait then rescan" program could be deemed the proper course [7]. Of contrary opinion is Brackmann et al. [23], regarding situations in which tumours are of less than $3 \mathrm{~cm}$ in diameter when early surgical management is recommended. It can preserve normal hearing in $30-65 \%$ and the functioning of facial nerves in $75-92 \%$ of patients. The role of stereotactic radiotherapy is not yet clearly determined [7].

The majority of meningiomas located in hemispheres and spinal canal can be resected safely and radically. In our patient, the location of the meningioma in the posterior cranial fossa made it impossible to remove completely. In this situation, we agreed that multi-variety protocols should be used. Due to chemotherapy, her overall survival was prolonged.

\section{Conclusions}

The prognosis in NF2 is still poor. The unification of management strategy in NF2 patients is mandatory in providing effective treatment. Integrated, multidisciplinary care consisting of a neurosurgeon, oncologist, otolaryngologist, neurologist, geneticist, ophthalmologist, pathologist and radiologist is necessary.

Open Access This article is distributed under the terms of the Creative Commons Attribution Noncommercial License which permits any noncommercial use, distribution, and reproduction in any medium, provided the original author(s) and source are credited.

\section{References}

1. Asthagiri AR, Parry DM, Butman JA et al (2009) Neurofibromatosis type 2. Lancet 373:1974-1986

2. Patronas NJ, Courcoutsakis N, Broley Ch M, Katzman GL, MacCollin M, ParryParry DM (2001) Intramedullary and spinal canal tumors in patients with neurofibromatosis 2; MR imaging findings and correlation with genotype. Radiology 218:434-442

3. Mautner VF, Baser ME, Thakkar SD et al (2002) Vestibular schwannoma growth in patients with neurofibromatosis type 2: a longitudinal study. J Neurosurg 96:223-228

4. Evans DG (2009) Neurofibromatosis type 2 (NF2): a clinical and molecular review. Orphanet J Rare Dis 19. doi:10.1186/ $1750-1172-4-16$
5. Harada H, Kumon Y, Hatta N, Sakaki S, Ohta S (1999) Neurofibromatosis type2 with multiple primary brain tumors in monozygotic twins. Surg Neurol 51:528-535

6. Janse AJ, Tan WF, Majoie ChBLM, Bijlsma EK (2001) Neurofibromatosis type 2 diagnosed in the absence of vestibular schwannomas. A case report and guidelines for a screening protocol for childrenat risk. Eur J Pediatr 160:439-443

7. Moffat DA, Quaranta N, Baguley DM, Hardy DG, Chang P (2003) Management strategies in neurofibromatosis type 2. Otology 260:12-18

8. Mautner VF, Baser ME, Thakkar SD, Feigen UM, Friedman JM, Kluwe L (2002) Vestibular schwannoma growth in patients with neurofobromatosos type 2: a longitudinal study. J Neurosurg 96:223-228

9. Reed N, Gutmann DH (2001) Tumorigenesis in neurofibromatosis: new insights and potential therapies. Trends Mol Med 4:157162

10. National Institutes of Health (1991) Acoustic neuroma. NIH Consensus Statement 9:1-24

11. Gutmann DH, Aylsworth A, Carey JC, Korf B, Marks J, Pyeritz RE, Rubenstein A, Viskochil D (1997) The diagnostic evaluation and multidisciplinary management of neurofibromatosis 1 and neurofibromatosis 2. JAMA 278:51-57

12. Mautner VF, Tatagiba M, Guthoff R, Samii M, Pulst SM (1993) Neurofibromatosis 2 in the pediatric age group. Neurosurgery 33:92-96

13. Nunes F, MacCollin M (2003) Neurofibromatosis 2 in the pediatric population. J Child Neurol 18:718-724

14. Evans DGR, Baser ME, O'Reilly B et al (2005) Management of the patient and family with neurofibromatosis 2: a consensus conference statement. Br J Neurosurg 1:5-12

15. Bosch MM, Boltshauser E, Harpes P, Landau K (2006) Ophthalmologic findings and long-term course in patients with neurofibromatosis type 2. Am J Ophthalmol 141:1068-1077

16. MacCollin M, Mautner VF (1998) The diagnosis and management of neurofibromatosis 2 in childhood. Semin Pediatr Neurol 5:243252

17. Dow G, Biggs N, Evans G, Gillespie J, Ramsden R, King A (2005) Spinal tumors in neurofibromatosos type 2. Is emerging knowledge of genotype predictive of natural history? J Neurosurg Spine 2:574-579

18. Ruggieri M, Iannetti P, Polizzi A et al (2005) Earliest clinical manifestations and natural history of neurofibramatosis type 2 (NF2) in childhood: a study of 24 patients. Neuropediatrics 36:21-34

19. Mautner VF, Tatagiba M, Lindenau M et al (1995) Spinal tumors in patients with neurofibramatosis type 2: MR imaging study of frequency, multiplicity and variety. AJR Am J Roentgenol 165:951-955

20. Pascual-Castroviejo I, Pascual-Pascual S, Viaño J (2009) Neurofibromatosis type 2 (NF2). Study of 7 patients. Neurologia 24:457-461

21. Fisher LM, Doherty JK, Lev NH, Slattery WH III (2007) Distribution of nonvestibular cranial nerve schwannomas in neurofibromatosis 2. Otol Neurotol 28:1083-1190

22. Perry A, Giannini C, Raghawan R et al (2001) Aggressive phenotypic and genotypic features in pediatric and NF2-associated meningiomas: a clinicopathologic study of 53 cases. J Neuropath Exp Neurol 60:994-1003

23. Brackmann DE, Fayad JN, Slattery WH et al (2001) Early proactive management of vestibular schwannomas in neurofibromatosis type 2. Neurosurgery 49:274-283 